\title{
Prevalencia de fibrilación auricular y factores relacionados en una población del centro de Madrid
}

\author{
F. J. CANDEL, M. MATESANZ, F. COGOLLUDO, I. CANDEL, C. MORA, \\ T. BESCOS, M. MARTÍN, I. VILA I COSTA
}

Servicio de Medicina Interna. Servicio de Cardiología. Hospital Clínico Universitario San Carlos. Centro de Salud de Espronceda. Madrid PREVALENCE OF ATRIAL FIBRILLATION AND RELATIONED FAC-
TORS IN A POPULATION IN THE CENTRE MADRID

\section{RESUMEN}

Introducción: La fibrilación auricular es la arritmia más frecuente en la práctica clínica y un importante factor de riesgo para ictus. El objetivo de nuestro estudio es conocer su prevalencia en un distrito central de Madrid.

Material y métodos: Se trata de un estudio retrospectivo, analizando historias clínicas procedentes de 13.945 pacientes del centro de atención primaria. A partir de éstas, se cumplimentó un protocolo clínico-epidemiológico. Tiempo de recogida de los datos: de septiembre de 2000 a mayo de 2001; tiempo de análisis: de septiembre de 2001 a mayo de 2002.

Resultados: Se obtuvieron un total de 425 fibrilaciones auriculares, 348 no valvulares y 77 valvulares. La prevalencia global de fibrilación auricular en pacientes mayores de 40 años fue de $2,52 \%$. Por sexos, fue de $2,47 \%$ en varones y $2,55 \%$ en mujeres. Los factores de riesgo más frecuentemente asociados a fibrilación auricular no valvular fueron la HTA, diabetes, cardiopatía isquémica y disfunción ventricular. En la valvular la HTA, el crecimiento auricular mayor de $45 \mathrm{~mm}$ y la disfunción ventricular.

Conclusiones: La fibrilación auricular presenta en la zona estudiada una prevalencia y distribución similar a la encontrada en otros países europeos.

PALABRAS CLAVE: Fibrilación auricular. Prevalencia.

\begin{abstract}
Introduction: Atrial fibrillation is the most common arrhythmia on clinical practice and an important risk factor for ictus. The aim of this study was to know the prevalence of this arrythmia in a central district of Madrid.

Material and methods: A retrospective study was done analysing medical records of 13.945 patients belonging to a primary care centre of Madrid. Time of study: recruitment of data from September 2000 to May 2001; analysis of data: from September 2001 to May 2002.

Results: We obtained a total of 425 atrial fibrillations, 348 non-valvular and 77 valvular. Global prevalence of atrial fibrillation in patients over 40 years in our area was $2.52 \%$. (2.47\% male, $2.55 \%$ female). Risk factors for atrial fibrillation more frequently found in non-valvular were high blood preassure (HBP), diabetes, ischaemic heart disease and left ventricular dysfunction. Among valvulars we found HBP, left atrial size over $45 \mathrm{~mm}$ and left ventricular dysfunction.

Conclusions: Atrial fibrillation shows a prevalence and distribution in the studied area similar to that found in other European countries.
\end{abstract}

KEY WORDS: Atrial fibrillation. Prevalence.

Candel FJ, Matesanz M, Cogolludo F, Candel I, Mora C, Bescos T, Martín M, Vila i Costa I. Prevalencia de fibrilación auricular y factores relacionados en una población del centro de Madrid. An Med Interna (Madrid) 2004; 21: 477-482.

\section{INTRODUCCIÓN}

La fibrilación auricular (FA) constituye la arritmia cardiaca más frecuente en la práctica clínica. Entre sus efectos indeseables figuran el aumento e irregularidad de la frecuencia ventricular, capaz de deparar signos de bajo gasto cardíaco o instauración de una insuficiencia cardiaca; la ausencia de contracción auricular, que puede ser importante en pacientes con afectación de la contractilidad ventricular (p. ej. miocardiopa- tías); y la presencia de embolias, sobre todo sistémicas, cuya profilaxis es continuo objeto de publicaciones en la comunidad medica internacional.

Datos obtenidos del Manitoba Follow Up Study (MFUS) (1), con un seguimiento de 44 años (1948-1992) arrojan una incidencia aproximada de 0,5 nuevos casos por cada 1.000 habitantes/año en menores de 50 años, 2,3 por 1.000 habitantes/año en la década de los 60 años, 9,7 por 1.000 habitantes/año en mayores de 70 y 16,9 por 1.000 habitantes/año en

Trabajo aceptado: 7 de mayo de 2004 
mayores de 85. Por tanto, hablamos de una enfermedad cuya prevalencia conviene conocer en nuestro entorno, en el que es creciente la población anciana.

Con objeto de conocer la prevalencia de FA en nuestro medio así como sus factores clínicos relacionados, ideamos un estudio transversal recabando información de las Historias Clínicas de los pacientes del Centro de Salud de Espronceda de Madrid. La recogida de datos se inició en septiembre de 2000 y el análisis en septiembre de 2001.

\section{PACIENTES Y MÉTODO}

Para estimar la prevalencia poblacional de FA en nuestra área se diseñó un estudio retrospectivo sobre una muestra representativa de la población a estudio (Zona Básica de Salud de Chamberí, Madrid). La Zona Básica de Salud (ZBS) de Chamberí en Madrid, tiene una población total de 147.229 personas (septiembre de 2000), de los cuales 70.207 son mayores de 40 años. Está atendida por 5 centros de atención primaria; el C. S. Espronceda (centro de nuestro estudio) tiene una población de 16.747 pacientes mayores de 40 años (23,85\% del área), 13.945 de ellos con Historia Clínica abierta. La población diana del estudio estuvo integrada por todas las personas mayores de 40 años censadas en la ZBS de Chamberí (70.207), mientras que la población a estudio o disponible estuvo integrada por los pacientes mayores de 40 años con historia abierta en cualquiera de los 16 cupos de Atención Primaria que constituye el C.S de Espronceda (13.945). El Criterio de Inclusión para nuestro estudio fue disponer de Historia Clínica abierta en el Centro y padecer FA (valvular o no valvular). Los Criterios de Exclusión fueron haber fallecido en el momento de recogida de los datos o tener menos de 40 años.

Para la selección de la muestra se establecieron 5 conglomerados (1 por cada Centro de Salud de la Zona Básica de Salud), siendo todos ellos homogéneos en cuanto a características demográficas y socio-sanitarias. El tamaño muestral se fijó aplicando 2 criterios: la disponibilidad de la información y los datos sobre prevalencia/incidencia de FA. La muestra se recogió en uno de los 5 conglomerados, tomando a todos los pacientes accesibles (aquellos con historia clínica abierta) con objeto de determinar la prevalencia de FA en nuestra población. Se revisaron finalmente 13.945 historias $(19,86 \%$ de la población diana). La prevalencia muestral de fibrilación auricular se calculó aplicando su definición epidemiológica como casos totales, incidentes ó no, entre el total de sujetos posibles. Posteriormente, se realizó una estimación por intervalo de la prevalencia poblacional a partir de la prevalencia muestral (inferencia estadística por intervalos). Para ello, se utilizó el método exacto para el cálculo de los límites de confianza de una proporción (variable binomial) mediante la distribución $F$ de Snedecor (recogida por Martín Andrés y Luna del Castillo) (2). La información existente en la literatura médica establece la prevalencia de la FA en la población general en torno al 26\%; el tamaño de nuestra muestra permitía detectar valores de prevalencia poblacionales en torno al $4 \%( \pm 2 \%)$, con un nivel de confianza del 99\%. El tipo de muestreo (un conglomerado al azar de los 5 que componen el área básica), y el tamaño muestral (19,86\% de la población diana) eran garantía de la representatividad de la muestra.

A todos los pacientes con FA se les cumplimentó un protocolo clinico-epidemiológico en el que se registraron edad, sexo y la presencia de los principales factores de riesgo relacionados con la FA (HTA, diabetes mellitus, disfunción ventricular, aurícula $>45 \mathrm{~mm}$, cardiopatía isquémica). Se consideró al paciente como portador de diabetes mellitus si constaban en la historia parámetros definitorios de la misma según los criterios de la American Diabetes Association (3). Así mismo se consideró al paciente portador de una HTA si en la historia constaban cifras tensionales definitorias de la misma según el JNC (4). Se consideró al paciente como portador de disfunción ventricular cuando en el informe ecocardiográfico de la historia clínica constaba este diagnóstico basado en criterios ecocardiográficos (Diámetro Telediastolico en Ventrículo Izquierdo (DTDVI) $\geq 55 \mathrm{~mm}$ ó Fracción de Eyección en Ventrículo Izquierdo (FEVI $\leq 45 \%$ ). El tamaño auricular se determinó también ecocardiograficamente en la proyección plano paraesternal, eje largo. La cardiopatía isquemica mediante constancia en la historia clinica de diagnóstico de esta enfermedad en base a parámetros clínicos, electro ó ecocardiográficos ó mediante un test de reserva coronaria positivo.

El trabajo de campo fue realizado en los meses de septiembre de 2000 y mayo de 2001, y el análisis de los datos entre septiembre de 2001 y mayo de 2002. La Base de Datos obtenida fue configurada en Microsoft ACCESS (Office 2000 para Windows). El tratamiento estadístico posterior se realizó utilizando EpiInfo 5.0 (en castellano) y SPSS vs 7.5 para Windows.

\section{RESULTADOS}

De las 16.747 personas asignadas al C. S. Espronceda, 13.945 tenían Historia Clínica abierta (población accesible). De ellos, 5.297 eran varones $(37,98 \%)$ y $8.648(62,01 \%)$ eran mujeres. Tras revisar las Historias Clínicas, se encontraron en total 425 casos de FA. Una vez aplicados los criterios de exclusión (es decir, rechazados los casos de pacientes fallecidos en el momento del estudio o menores de 40 años), finalmente se obtuvieron 352 casos de fibrilación auricular (221 mujeres y 131 varones) útiles para el cálculo de la prevalencia en nuestro estudio.

La prevalencia total de FA en nuestra muestra fue de $2,52 \%$. Por sexos, la prevalencia en varones fue de $2,47 \%$, y de $2,55 \%$ en mujeres. Para inferir la prevalencia poblacional a partir de la prevalencia muestral se utilizó el método exacto para el cálculo de los límites de confianza de una proporción (variable binomial) mediante la distribución $\mathrm{F}$ de Snedecor. La prevalencia poblacional se estimó en 2,52\% (intervalo de confianza entre 1,582 y 4,013, calculado para el $99 \%$ de confianza y 120 grados de libertad). Por sexos la prevalencia poblacional de FA se estimó en 1,60-4,07 para mujeres y $1,55-3,95$ para varones.

Se realizó un análisis descriptivo sobre los 425 casos totales detectados de FA en la muestra. De ellos, 348 fueron diagnosticados de FA no valvular (FANV) y 77 de FA valvular $(F A V)$. Del primer grupo (no valvular), 280 casos eran FA crónica y 68 FA paroxística. Del segundo grupo (valvular) 68 eran crónicas y 9 paroxísticas. La edad media al diagnóstico de las FANV fue de 75 años, con una desviación típica de 9 años; de ellas 127 (36,5\%) eran varones y $221(63,5 \%)$ eran mujeres. Entre los casos de FAV la edad media fue 67 años, con una desviación típica de 11 años. En este grupo se encontraron 21 varones $(27,3 \%)$ y 56 mujeres $(72,1 \%)$. 
La distribución de los casos de FA encontrados según décadas se muestra en la tabla I. La máxima incidencia en el diagnóstico estuvo entre los 70-79 años. Las características clínicas que más se repitieron en los pacientes con FA se exponen en la tabla II. mática sigue siendo la causa más frecuente de FA en Etiopía (15). Las prevalencias por países (5-7,9,16-28), según los estudios evacuados a la comunidad médica internacional se recogen en la tabla III. Los estudios en poblaciones de Europa y EE.UU. muestran una prevalencia similar de FA. Sin

TABLA I

DISTRIBUCIÓN DE LOS CASOS DE FA DETECTADOS SEGÚN GRUPOS DE EDAD

\begin{tabular}{lcccccc}
\hline & $40-49$ años & $50-59$ años & $60-69$ años & $70-79$ años & $80-89$ años & 90 años o más \\
\hline FANV & 5 & 13 & 60 & 135 & 115 & 20 \\
$(n=348)$ & $(1,4 \%)$ & $(3,7 \%)$ & $(17,2 \%)$ & $(38,8 \%)$ & $(33 \%)$ & $(5,7 \%)$ \\
FAV & 7 & 10 & 23 & 25 & 11 & 1 \\
$(n=77)$ & $(9,1 \%)$ & $(13 \%)$ & $(29,9 \%)$ & $(32,5 \%)$ & $(14,3 \%)$ & $(1,3 \%)$ \\
\hline
\end{tabular}

FANV:- fibrilación auricular no valvular; FAV:-fibrilación auricular valvular.

TABLA II

CARACTERÍSTICAS CLÍNICAS DE LA POBLACIÓN CON FA ESTUDIADA

\begin{tabular}{lccccc}
\hline & $\begin{array}{c}\text { Aurícula } \\
>45 \mathrm{~mm}\end{array}$ & HTA & $\begin{array}{c}\text { Diabetes } \\
\text { Mellitus }\end{array}$ & $\begin{array}{c}\text { Cardiopatía } \\
\text { isquémica }\end{array}$ & $\begin{array}{c}\text { Disfunción } \\
\text { ventricular Izda }\end{array}$ \\
\hline FANV & 35 & 208 & 83 & 73 & 90 \\
$(\mathrm{n}=348)$ & $(10,1 \%)$ & $(59,81 \%)$ & $(23,9 \%)$ & $(21 \%)$ & $(25,9 \%)$ \\
FAV & 23 & 24 & 10 & 10 & 21 \\
$(\mathrm{n}=77)$ & $(29,9 \%)$ & $(31,2 \%)$ & $(13,0 \%)$ & $(13,0 \%)$ & $(27,3 \%)$ \\
\hline
\end{tabular}

FANV:- fibrilación auricular no valvular; FAV:-fibrilación auricular valvular; HTA: Hipertensión arterial.

\section{DISCUSIÓN}

Según datos tomados de los cuatro estudios con mayor tamaño muestral (Cardiovascular Health Study [CHS] (5), Framingham Heart Study [FHS] (6), Western Australia Study (7) y Rochester MN Study (8)), la prevalencia global de la FA en la población general se encuentra en torno al 1,5-6,2\% (9). Por grupos etarios es, aproximadamente, del 0,2-0,3\% en 2535 años, 3-4\% en 55-60 años y 5-9\% en mayores de 65 años, llegando al $10 \%$ en la década de los 80 (9-12). Por sexos está en torno al $2,8 \%$ en mujeres y 5,9\% en varones entre 65-69 años, llegando a $6,7 \%$ en mujeres y $8,0 \%$ en varones de 80 ó más años (datos del CHS3). Aunque la probabilidad de padecer una FA es 1,5 veces mayor en hombres que en mujeres, la mayor supervivencia de éstas hace que cuenten con la mayor cantidad de casos de FA (53\%) (9).

La mayoría de la población de nuestro estudio pertenece a la raza caucásica. En el CHS el 5\% de la cohorte era afroamericana y en ésta existía una clara tendencia a la baja en la prevalencia de FA con respecto a la raza blanca ( RR 0,47, con un intervalo de confianza de 0,22-1,01) (13). También existen diferencias raciales entre los factores de riesgo que desencadenan la FA. En un estudio hospitalario inglés, el factor de riesgo más importante para la FA en población negra y afrocaribeña era la HTA, mientras que en los pacientes de origen asiático era la cardiopatía isquémica (14). La cardiopatía reu- embargo, en los estudios realizados en blancos y otros grupos étnicos mostraron que la prevalencia de FA en los primeros era del 2,4\% mientras que la cifra se reducía al 0,6\% en sudasiáticos de la misma $(24,25)$.

La prevalencia global de la FA en nuestro estudio del C.S Espronceda en mayores de 40 años, extrapolable a toda la zona de salud de Chamberí, fue de 2,524\%, con unos límites de confianza del $99 \%$ de 1,582 y 4,013\%. Los valores obtenidos son similares a los de Philips y cols. (8) en Rochester (MN, USA), a los de Ott y cols. (19) en Rotterdam (Holanda) y a los de Lip y cols. (24) en Birmingham (UK), algo menores que los de Furberg y cols. (5) (CHS) que los cifran en torno al $6,2 \%$ en varones y $4,8 \%$ en mujeres ó los de Sudlow y cols. (21) en Northumberland (UK) que se encuentran en torno al $4,7 \%$. Recientemente han sido publicados los resultados del estudio CARDIOTENS por García-Acuña y cols. (29) con una muestra de 32.051 pacientes y que cifraba la prevalencia global de FA en $2,75 \%$.

La prevalencia por sexos fue de $2,473 \%$ en varones, con un intervalo de confianza de 1,55 y 3,95 ; y para mujeres de $2,555 \%$ con un intervalo de confianza de 1,602 y 4,07 . La prevalencia por sexos en todos los estudios suele ser mayor en el varón, y de hecho, el riesgo de padecerla en éste es 1,5 veces el de la mujer (aunque dado que la supervivencia suele ser mayor en las mujeres, la enfermedad aparece más frecuentemente diagnosticada en ellas). No obstante, nuestra población 
TABLA III

PREVALENCIA DE FA EN DISTINTOS PAÍSES (MODIFICADA DE RYDER ET AL (7))

\begin{tabular}{|c|c|c|c|c|}
\hline Estudio & Pais & Edad & & \\
\hline Wolf y cols. (FHS) (6) & EE.UU. & $\geq 50$ & $\begin{array}{l}50-59 \\
60-69 \\
70-79 \\
80-89\end{array}$ & $\begin{array}{l}0,5 \% \\
1,8 \% \\
4,8 \% \\
8,8 \%\end{array}$ \\
\hline Furgberg y cols. (CHS) (5) & EE.UU. & $\geq 65$ & $\begin{array}{l}\text { Global } \\
65-69 \\
70-90 \\
>80\end{array}$ & $\begin{array}{l}6,2 \% \vee \text { y } 4,8 \% M \\
5,9 \% \vee \text { y } 2,8 \% M \\
5,8 \% \vee \text { y } 5,9 \% M \\
8,0 \% \vee \text { y } 6,7 \% M\end{array}$ \\
\hline Philips y cols. (8) & Rochester MN (USA) & $\geq 35$ & $\begin{array}{l}\text { Global } \\
25-34 \\
45-54 \\
55-64 \\
65-74 \\
>75\end{array}$ & $\begin{array}{l}2,8 \% \\
0,0 \% \\
0,5 \% \vee \text { y } 0,5 \% M \\
1,0 \% \vee \text { y } 1,5 \% M \\
6,0 \% \vee \text { y } 3,0 \% M \\
16,1 \% \text { y } 12,2 \% M\end{array}$ \\
\hline Boysen y cols. (12) & Copenhagen (Denmark) & $\geq 35$ & Global & $0,6 \%$ \\
\hline Onundarson y cols. (17) & Reyjavik (Iceland) & $32-64$ & Global & $0,41 \% \vee$ y $0,15 \% M$ \\
\hline Gehring y cols. (18) & West Germany & $25-64$ & Global & $0,22 \% \vee$ y $0,34 \% M$ \\
\hline Ott y cols. (19) & Rotterdam (Netherlands) & $55-106$ & $\begin{array}{l}\text { Global } \\
55 \\
65 \\
75 \\
\geq 85\end{array}$ & $\begin{array}{l}3,3 \% \vee \text { y } 2,7 \% M \\
0,9 \% \vee \text { y } 0,3 \% M \\
3 \% \vee \text { y } 1,8 \% M \\
7,6 \% \vee \text { y } 5,2 \% M \\
13,2 \% \vee \text { y } 10,6 \% M\end{array}$ \\
\hline Langenberg y cols. (20) & Netherlands & $\geq 60$ & $\begin{array}{l}\text { Global } \\
60-69 \\
70-79 \\
\geq 80\end{array}$ & $\begin{array}{l}5.1 \% \\
3,3 \% \vee \text { y } 2,3 \% M \\
7,0 \% \vee \text { y } 6,3 \% M \\
12,1 \% \vee \text { y } 8,7 \% M\end{array}$ \\
\hline Sudlow y cols. (21) & Northumberland (UK) & $\geq 65$ & $\begin{array}{l}>65 \\
\geq 75\end{array}$ & $\begin{array}{l}4,7 \% \\
10 \% \vee \text { y } 5,6 \% M\end{array}$ \\
\hline Wheeldon y cols. (22) & Sheffield (UK) & $\geq 65$ & $\begin{array}{l}\text { Global } \\
65-69 \\
70-74 \\
75-79 \\
80-84 \\
185\end{array}$ & $\begin{array}{l}5,4 \% \\
2,3 \% \\
4,1 \% \\
5,8 \% \\
6,4 \% \\
8,1 \%\end{array}$ \\
\hline Hill y cols. (23) & Staffordshire (UK) & $>65$ & $\begin{array}{l}\text { Global } \\
65-69 \\
70-74 \\
75-79 \\
80-84 \\
\geq 85\end{array}$ & $\begin{array}{l}3,7 \% \\
3,4 \% \\
2,7 \% \\
3,4 \% \\
5,4 \% \\
5,9 \%\end{array}$ \\
\hline Lip y cols. (24) & Birmingham (UK) & $>50$ & Global & $2,4 \%$ \\
\hline Lip y cols. (25) & $\begin{array}{l}\text { Birmingham (UK) } \\
\text { Indoasiaticos }\end{array}$ & $>50$ & Global & $0,6 \%$ \\
\hline Nakayama y cols. (26) & Shibata (Japan) & $\geq 40$ & Global & $1,3 \%$ \\
\hline Lak y cols. (27) & Hong-Kong (China) & $60-94$ & Global & $1,3 \%$ \\
\hline Kaushal y cols. (28) & Himalaya (India) & $>15$ & Global & $0,1 \%$ \\
\hline Lake y cols. (7) & Busselton, W (Australia) & $>60$ & $\begin{array}{l}\text { Global } \\
60-64 \\
65-69 \\
70-74 \\
\geq 75\end{array}$ & $\begin{array}{l}1,5 \% \\
1,1 \% \vee \text { y } 2,3 \% M \\
3,3 \% \vee \text { y } 2,7 \% M \\
8,6 \% \vee \text { y } 5,5 \% M \\
15 \% \vee \text { y } 11,6 \% M\end{array}$ \\
\hline García-Acuña y cols. (29) & Multicentrico (Spain) & $>50$ & $\begin{array}{l}\text { Global } \\
50-59 \\
60-69 \\
70-79 \\
>79 \\
\text { Global }\end{array}$ & $\begin{array}{l}2,75 \% \\
2,03 \% \\
3,41 \% \\
5,51 \% \\
8,26 \% \\
2,52 \%\end{array}$ \\
\hline
\end{tabular}


constaba de un $38 \%$ de varones y un $62 \%$ de mujeres, si los porcentajes hubieran sido del $50 \%$, las prevalencias habrían resultado mayores en varones, aunque no 1,5 veces (se habrían obtenido entonces valores de $3,15 \%$ en varones y $2,05 \%$ en mujeres).

La edad media de los pacientes con FA en nuestro estudio fue de 75 años en las FANV y 67 años en las FAV. La frecuencia acumulada se mostró máxima en el intervalo correspondiente a la séptima década de la vida $(38,8 \%$ en FANV y $32,5 \%$ en FAV). La FAV etiopatogénicamente aparece y da síntomas antes que la FANV; en su mayoría son pacientes con valvulopatía mitral reumática en la juventud que expresan los primeros síntomas de afectación valvular en la década de los 40, y con la evolución de la valvulopatía aumenta el tamaño auricular y aparece la arritmia. Este mecanismo suele suceder antes que el de la FANV, cuya etiopatogenia se relaciona más con la proliferación progresiva de celulas musculares lisas, fibras elásticas y colágeno en el endocardio auricular, aumento del tejido adiposo, fibrosis en el nodo sinusal e infiltración amiloide de todo el tejido auricular (30), y su aparición suele ser más tardía. Quizá por eso la edad media al diagnóstico de los pacientes con FANV fue de 75 años y la de los pacientes con FAV de 67. Sin embargo, la evolución de ambas se solapa en el tiempo, siendo en ambos tipos, máxima en el intervalo correspondiente a la séptima década de la vida.

Las características clínico-ecocardiográficas que más se repitieron en la FAV fueron la aurícula mayor de $45 \mathrm{~mm}$ $(29,9 \%)$, la disfunción ventricular $(27,3 \%)$ y la HTA $(31,2 \%)$. De éstos, los 2 primeros se encontrarían en relación con su etiopatogénia y fisiopatología (valvulopatía, dilatación auri- cular, insuficiencia cardíaca). También en el caso de las FANV las características clinicas que más se repitieron fueron la HTA $(59,81 \%)$, la diabetes mellitus $(23,9 \%)$, la cardiopatía isquémica $(21 \%)$ y la disfunción ventricular $(25,9 \%)$. Los 3 primeros son importantes factores de riesgo cardiovascular y aterosclerótico $(31,32)$. Aunque la HTA pudiera tener mayor identidad como factor predisponente para la FANV por su implicación en la etiopatogenia y fisiopatología de la aterosclerosis, que parece ser el sustrato para la producción de este tipo de arritmia, también aparece en una elevada proporción de enfermos con FAV. La explicación pudiera estar en la elevada prevalencia de la HTA en la población general, y más en la población añosa.

En conclusión, la FA es una enfermedad prevalente en nuestro entorno $(2,524 \%$; IC 1,582-4,013\%) cuyo interés radica fundamentalmente en sus complicaciones (precipitación de episodios de insuficiencia cardiaca, riesgo embólico, etc.). Son necesarios más estudios de prevalencia en nuestro medio con objeto de conocer si las diferencias con las otras series son debidas a la mayor o menor prevalencia de factores de riesgo cardiovascular, a factores ambientales, etc.

\section{LIMITACIONES DEL ESTUDIO}

Como consecuencia del diseño del estudio no se ha podido correlacionar el tipo ni el grado de severidad de la lesión valvular con la presencia o no de FA ni tampoco la fracción de eyección o la clase funcional de la NYHA con la existencia o no de FA.

\section{Bibliografía}

1. Khran AD, Manfreda J, Tate RB, Mathewson FA, Cuddy TE. The natural history of atrial fibrillation: incidence, risk factors and prognosis in the Manitoba follow-up study. Am J Med 1995; 98: 476-84.

2. Martín Andrés A, Luna del Castillo JD. Bioestadística para las ciencias de la Salud. $2^{a}$ Ed. Madrid: Ed Norma. 1989.

3. Management of Diabetes. American Diabetes Association. Diabetes Care 2000; 23 (Supl. 1).

4. The fifth report of the Joint National Committee on Detection, Evaluation, and Treatment of High Blood Pressure (JNC V). Arch Intern Med 1993; 153 (2): 154-83.

5. Furberg CD, Psaty BM, Manolio TA, Gardin JM, Smith VE, Rautaharju PM. for the CHS collaborative research group. Prevalence of atrial fibrillation in elderly subjects (the Cardiovascular Health Study). Am J Cardiol 1994; 74: 236-41.

6. Wolf PA, Abbott RD, Kannel WB. Atrial fibrillation as an independent risk factor for stroke: the Framingham heart study. Stroke 1991; 22: 983-8.

7. Lake FR, Cullen KJ, de Klerk NH, Mc Call MG, Rosman DL. Atrial fibrillation and mortality in an elderly population Aust NZ J Med 1989; 19: 321-6 in Ryder, KM; Benjamin, EJ. Epidemiology and significance of atrial fibrillation. Am J Cardiol 1999; 84: 131R-8R.

8. Philips SJ, Whisnant JP; O'Fallon WM, Frye RL.Prevalence of cardiovascular disease and diabetes mellitus in residents of Rochester, Minnesota Mayo Clin Proc 1990; 65: 344-59.

9. Ryder KM, Benjamin EJ. Epidemiology and significance of atrial fibrillation. Am J Cardiol 1999; 84: 131R-8R.

10. Pobrid PJ. Atrial Fibrillation in the elderly. Cardiol Clin 1999; 17 (1): 173-88.

11. Levy S, Breithardt G, Campbell RWF, Camm AJ, Daubert JC, Allesie $\mathrm{M}$, et al Atrial fibrillation current knowledge and recomendations for management (working group report). Eur Heart J 1998; 19: 1294-320.
12. Benjamin EJ, Levy D, Varizi SM, D'Agostino RB, Belanger AJ, Wolf PA. Independent risk factors for atrial fibrillation in a population-based cohort. The Framingham heart study. JAMA 1994; 271 (4): 840-4.

13. Psaty BM, Manolio TA, Kuller LH, Kronmal RA, Cushman M, Fried LP, et al Incidence of and risk factors for atrial fibrillation in older adults. Circulation 1997; 96: 2455-61.

14. Zafiris J, Beevers DG, Lip GYH. Acute admissions with atrial fibrillation in a British multirracial hospital. Br J Clin Prac 1997; 51: 91-6 in Ryder, KM; Benjamin, EJ. Epidemiology and significance of atrial fibrillation. Am J Cardiol 1999; 84: 131R-8R.

15. Maru M. Atrial Fibrillation and embolic complications. E Afr Med J 1997; 74: 3-5 in Ryder, KM; Benjamin, EJ. Epidemiology and significance of atrial fibrillation. Am J Cardiol 1999; 84: 131R-8R.

16. Boysen G, Nyboe J, Appleyard M, Sorensen PS, Boas J, Somnier F, et al.Stroke incidence and risk factors for stroke in Copenhagen, Denmark. Stroke 1988; 19: 1345-53.

17. Onundarson PT, Thorgeirsson G, Jonmundsson E, Sigfusson N, Hardarson T. Chronic atrial fibrillation-epidemiologic features and 14 years follow-up: a case-control study. Eur Heart J 1987; 8: 521-7.

18. Gehring J, Perz S, Stieber J, Kufner R, Keil U. Cardiovascular risk factors, ECG abnormalities and quality of life in subjects with atrial fibrillation. Soz Praventivmed 1996; 41: 185-93 in Ryder, KM; Benjamin, EJ. Epidemiology and significance of atrial fibrillation. Am J Cardiol 1999; 84: 131R-8R.

19. Ott AO, Breteler MMB, de Bruyne MC, van Harskamp F, Grobbee DE, Hoffman A. Atrial fibrillation and dementia in a population-based study: the Rotterdam study. Stroke 1997; 28: 316-21.

20. Langenberg M, Hellemons BSP, van Ree JW, Vermeer F, Lodder J, Schouten HJA, et al. Atrial fibrillation in elderly patients: prevalence and comorbidity in general practice. BMJ 1996; 313: 1534. 
21. Sudlow M, Thompson R, Thwaites B, Rodgers H, Kenny RA. Prevalence of atrial fibrillation and elegibility for anticoagulation in the comunity. Lancet 1998; 352: 1167-71

22. Wheeldom NM, Tayler DI, Anagnostou E, Cook D, Wales C, Oakley GDG. Screening for atrial fibrillation in primary care. Heart 1998; 79: 50-5.

23. Hill JD, Mottram EM, Killeen PD. Study of the prevalence of atrial fibrillation in general practice patients over 65 years of age. J R Coll Gen Pract 1987; 37: 172-3.

24. Lip GYH, Golding DJ, Nazir M, Beevers DG, Child DL, Fletcher RI. A survey of atrial fibrillation in general practice: the west Birmingham atrial fibrillation proyect. Br J Gen Pract 1997; 47: 285-9.

25. Lip GYH, Bawden L, Hodson R, Rutland E, Snatchfold J, Beevers DG. Atrial fibrillation among the Indo-Asian general practice population: the west Birmingham atrial fibrillation proyect. Int J Cardiol 1998; 65: 187 92.

26. Nakayama T, Date C, Yokohama T, Yoshiike N, Yamaguchi M, Tanaka H. A 15.5 years follow-up study of stroke in a japanese provincial city. Stroke $1997 ; 28 ; 45-52$.
27. Lak NS, Lau CP. Prevalence of palpitations, cardiac arrhythmias and their associated risk factors in ambulatory elderly. Int J Cardiol 1996; 54: 231-6.

28. Kaushal SS, DasGupta DJ, Prashar BS, Bhardwaj AK. Electrocardiographic manifestation of healthy residents of a tribal Hymalayan village. J Assoc Physicians India 1995; 43: 15-16 in Ryder, KM; Benjamin, EJ. Epidemiology and significance of atrial fibrillation. Am J Cardiol 1999; 84: 131R-8R.

29. García-Acuña JM, González-Juanatey JR, Alegría E, González I, Listerri JL. La fibrilación auricular permanente en las enfermedades cardiovasculares en España. Estudio CARDIOTENS 1999. Rev Esp Cardiol 2002; 55: 943-52.

30. Falk RH. Etiology and complications of atrial fibrillation: insights from pathology studies. Am J Cardiol 1998; 82: 10N-17N.

31. Pyörälä K, De Backer Y, Graham P, et al. Prevention of coronary heart disease in clinical practice. Recommendations of the task force of the European Society of Cardiology, European Atherosclerosis Society and European Society of Hypertension. Eur Heart J 1994; 15: 1300-31.

32. Anderson KM, Wilson PWF, Odell PM, et al. An Updated Coronary Risk Profile. A Statement for Health Proffessional. Circulation 1991; 83: 356-62. 